\title{
From Hubble diagrams to scale factors
}

\author{
T. Schücker ${ }^{1, \star, \star \star}$ and A. Tilquin ${ }^{2, \star \star \star}$ \\ 1 Centre de Physique Théorique, CNRS - Luminy, Case 907, 13288 Marseille Cedex 9, France \\ e-mail: schucker@cpt.univ-mrs.fr \\ 2 Centre de Physique des Particules de Marseille, CNRS - Luminy, Case 907, 13288 Marseille Cedex 9, France \\ e-mail: tilquin@cppm.in2p3.fr
}

Received 21 June 2005 / Accepted 23 September 2005

\section{ABSTRACT}

Aims. We study the image of the transform from scale parameters to Hubble diagrams and present a lower bound on the radius of the universe today $a_{0}$ and a monotonicity constraint on the Hubble diagram.

Methods. Our theoretical input is minimal: Einstein's kinematics and maximally symmetric universes.

Results. Present supernova data yield $a_{0}>1.2 \times 10^{26}$, i.e. $\Omega_{k 0}>-1.29$. We attempt to quantify the monotonicity constraint and do not see any indication of non-monotonicity.

Key words. cosmological parameters

\section{Introduction}

In a homogeneous, isotropic, expanding universe, the apparent luminosity of a standard candle is a monotonically decreasing function of the time of flight of emitted photons if the universe is open. This is also true in spherical universes if the time of flight is small enough with respect to the radius divided by the speed of light. In principle, the (apparent) luminosity $\ell(t)$ as a function of time can be used to measure the scale factor $a(t)$. In reality, arriving photons do not tell us their time of flight, but only their spectral deformation, $z:=v_{\text {table }} / v_{\text {observed }}-1$, where $v$ is the frequency of the photon. In an expanding universe $z$ is positive, "red shift". If we pretend to know the scale factor $a(t)$, we can compute the luminosity $\ell(z)$ and confront it with the Hubble diagram. The transform $a(t) \rightarrow \ell(z)$ reminds us of the Fourier transform and of course we are interested in the inverse transform $\ell(z) \rightarrow a(t)$. Therefore we must ask three questions: what is the domain of definition of the initial transform, what is its image, and is the transform injective? Also, should the measured luminosity $\ell(z)$ be "far away" from the image our working hypotheses are made to test.

* Unité Mixte de Recherche (UMR 6207) du CNRS et des Universités Aix-Marseille 1 et 2 et Sud Toulon-Var, Laboratoire affilié à la FRUMAM (FR 2291), France.

$\star \star$ Also at Université de Provence, France.

$\star \star \star$ Unité Mixte de Recherche (UMR 6550) du CNRS et de l’Université Aix-Marseille 2, France.

\section{The hypotheses}

We assume the kinematics of general relativity:

- The gravitational field is coded in a time-space metric of signature + - - and the configuration space is the set of all such metrics.

- Point-like test particles, massive or massless, which are only subject to gravity, follow time-like or light-like geodesics.

- Point-like clocks, e.g. atomic clocks, are necessarily massive. They move on time-like curves (not necessarily geodesics) and indicate proper time $\tau$.

This kinematics is covariant under general coordinate transformations. In 1983 the meter was officially abandoned as a fundamental unit in favor of an absolute speed of light, and at least since then, any non-covariant kinematics is void of any physical meaning.

We also assume the comological hypotheses of maximally symmetric spaces:

- The metric is Robertson-Walker,

$c^{2} \mathrm{~d} \tau^{2}=c^{2} \mathrm{~d} t^{2}-a(t)^{2}\left[\mathrm{~d} \boldsymbol{x}^{2}+k \frac{(\boldsymbol{x} \cdot \mathrm{d} \boldsymbol{x})^{2}}{1-k \boldsymbol{x}^{2}}\right]$.

The scale factor $a(t)$ is a strictly positive function of time, $k=-1,0,1$ for the pseudosphere, the Euclidean space, and the sphere. We take the coordinates $\boldsymbol{x}$ as dimensionless and call them "co-moving position", while the scale 
factor is measured in meters. For closed universes, $k=1$, we must restrict the spatial coordinates to the unit ball, $|\boldsymbol{x}|<1$, which describe the northern hemisphere. For open universes, $\boldsymbol{x}$ varies in $\mathbb{R}^{3}$.

- The test particles are (superclusters of) galaxies and photons. The former follow the time-like geodesics $t=\tau, \boldsymbol{x}=$ const. Note that, due to the high degree of symmetry, the proper time is universal for all these time-like geodesics and is taken as the time coordinate. The photons are emitted from a galaxy at time $t$ and arrive at our position at time $t_{0}$, today.

The symmetry hypothesis and the choice of the coordinates $(t, \boldsymbol{x})$ have reduced the configuration space to the set of positive functions $a(t)$ on the real line and to the parameter $k=0, \pm 1$.

\section{The Hubble diagram}

From these hypotheses we can compute (see for instance Berry 1976) the (apparent) luminosity $\ell(t)$ of a standard candle in Watt $/ \mathrm{m}^{2}$ as a function of emission time $t$

$\ell(t)=\frac{L}{4 \pi a_{0}^{2}} \frac{a(t)^{2}}{a_{0}^{2} s^{2}(\chi(t))}$

where $L$ is the absolute luminosity of the standard candle in Watt, $a_{0}:=a\left(t_{0}\right)$ is the scale factor today;

$\chi(t):=\int_{t}^{t_{0}} \frac{c \mathrm{~d} \tilde{t}}{a(\tilde{t})}$

is the dimensionless co-moving geodesic distance covered by the photon; and

$s(\chi):=\left\{\begin{array}{cc}\sin \chi, & k=1 \\ \chi, & k=0 \\ \sinh \chi, & k=-1 .\end{array}\right.$

The luminosity has a singularity at $t=t_{0}$, called the "short distance divergency", for any value of $k$ and any scale factor. For closed universes, $k=1$ we may have additional singularities for $\chi=\pi$, called the "antipode divergencies", while for $\chi=2 \pi$ we are back at a second short distance divergency, and so forth if $\chi$ continues to grow. Of course there might be a horizon, i.e. an upper bound for $\chi$, masking all or some of the singularities.

From the above hypotheses, we can also compute the spectral deformation as a function of emission time,

$z(t)=\frac{a_{0}}{a(t)}-1$

The theoretical Hubble diagram is the parametric plot in the $z-\ell$ plane as $t$ varies.

If we suppose that the scale factor is strictly increasing with $\dot{a}:=\mathrm{d} a / \mathrm{d} t>0$, then $z$ is positive, "red shift", and we can invert the function $z(t)$. For convenience, we write $t(z)$ for its inverse. Then the Hubble diagram is a function $\ell(t(z))$ that, still for convenience, is written $\ell(z)$,

$\ell(z)=\frac{L}{4 \pi a_{0}^{2}} \frac{1}{(z+1)^{2} s^{2}(\chi(z))}$ where

$\chi(z):=\chi(t(z))=\frac{c}{a_{0}} \int_{0}^{z} \frac{\mathrm{d} \tilde{z}}{H(\tilde{z})}$

and $H(z):=\dot{a}(t(z)) / a(t(z))$ is the Hubble rate.

The short distance divergency now is at $z=0$ and easy to get rid of. Let us define the regularized luminosity

$f(z):=z^{2} \ell(z)=\frac{L}{4 \pi a_{0}^{2}} \frac{z^{2}}{(z+1)^{2} s^{2}(\chi(z))}$.

Indeed,

$f(0)=\frac{L}{4 \pi c^{2}} H_{0}^{2}, \quad f^{\prime}(0)=\frac{L}{4 \pi c^{2}} H_{0}^{2}\left(q_{0}-1\right)$,

where the prime is differentiating with respect to $z, q(z)$ is the deceleration parameter,

$q(z)=-\frac{a \ddot{a}}{\dot{a} \dot{a}}(t(z))$,

and $H_{0}:=H(0), q_{0}=q(0)$.

\section{The transform $a(t) \longrightarrow \ell(z)$}

Let us first try to describe the image, that is, all luminosity functions $\ell(z)$ that can be obtained from strictly increasing scale factors $a(t)$ with $\dot{a}>0$ and with $k=0, \pm 1$.

We already know that $\ell(z)$ comes with the short distance divergency at $z=0$, which is such that $z^{2} \ell(z)=: f(z)$ is regular there. For open universes, there are no other singularities. In fact, $(z+1)^{2} \ell(z)=: g(z)$ is a decreasing function. For closed universes, on the other hand, $g(z)$ goes through a minimum as the photons pass the equator, $\chi=\pi / 2$. From there on, the luminosity increases again and goes to the antipode divergency. It might happen, of course, that the equator is masked by the horizon, in which case $g(z)$ remains decreasing forever, even though the universe is closed.

Let us now ask whether the transform is injective in the domain of increasing scale factors. If we pretend to know $k$, the answer is affirmative for open universes. Indeed, solving Eq. (8) for $s(\chi)$ and differentiating with respect to $z$ yields (Esposito-Farèse \& Polarski 2001)

$$
\begin{aligned}
\frac{a_{0}}{c} H(z) & =\frac{(z+1) \sqrt{4 \pi a_{0}^{2} L^{-1}(z+1)^{2} f(z)-k z^{2}}}{1-\frac{1}{2} z(z+1) f^{\prime}(z) / f(z)} \\
& =\frac{z(z+1) \sqrt{4 \pi a_{0}^{2} L^{-1} g(z)-k}}{1-\frac{1}{2} z(z+1) f^{\prime}(z) / f(z)}
\end{aligned}
$$

Therefore we can reconstruct the Hubble rate from the luminosity.

Integrating the Hubble rate with respect to $t$ gives us the scale factor with the ambiguity of the initial condition $a_{0}$. But for flat universes, this initial condition is unphysical. By a coordinate transformation of $\boldsymbol{x}$, we can set $a_{0}=1 \mathrm{~m}$. This is different for curved universes, where $a_{0}$ is related to a local observable, the curvature. In the closed case, $a_{0}$ is also related to a global observable, the radius of the universe today. However, 
unless $g(z)$ already exhibits an increase, only a lower bound on the radius today can be reconstructed from the luminosity,

$a_{0} \geq \sqrt{\frac{L}{4 \pi g_{\min }}}, \quad g_{\min }:=\min _{z>0}\left\{(z+1)^{2} \ell(z)\right\}$.

Note that this lower bound does not depend on the absolute luminosity $L$.

If we admit that we do not know $k$ and if the luminosity function $\ell(z)$ satisfies $(i) z^{2} \ell(z)$ is regular at $z=0$; and (ii) $(z+1)^{2} \ell(z)$ is decreasing, then there are three positive functions $a_{ \pm}(t)$ and $a(t)$, such that the universes with scale factor $a_{-}(t), k=-1$, with scale factor $a(t), k=0$ and with scale factors $a_{+}(t), k=1$ have the same luminosity function $\ell(z)$. These three scale factors satisfy

$a_{-}\left(t_{0}\right) \sinh \chi_{-}=a\left(t_{0}\right) \chi=a_{+}\left(t_{0}\right) \sin \chi_{+}$.

Note that in the flat case, the "initial condition" $a\left(t_{0}\right)$ carries no information, while in the closed case $a_{+}\left(t_{0}\right)$ must satisfy the inequality (12).

Example (constant deceleration parameter):

take the scale factor,

$a(t):=a_{0}\left(p H_{0} t\right)^{1 / p}, \quad p>0, \quad 0<t<t_{0}=\frac{1}{p H_{0}}$.

Then the Hubble rate is $H=H_{0}(z+1)^{p}$ and the deceleration parameter is constant, $q \equiv p-1$. The co-moving distance is

$\chi=\frac{c}{a_{0} H_{0}} \ln (z+1)=-\frac{c}{a_{0} H_{0}} \ln \left(H_{0} t\right), \quad p=1$,

$$
\begin{aligned}
\chi & =\frac{c}{(p-1) a_{0} H_{0}}\left(1-(z+1)^{1-p}\right) \\
& =\frac{c}{(p-1) a_{0} H_{0}}\left(1-\left(p H_{0} t\right)^{1-1 / p}\right), \quad p \neq 1 .
\end{aligned}
$$

For $p>1$, there is a horizon at $\chi=c /\left((p-1) a_{0} H_{0}\right)$. In particular, if $p=2$, and $k=0$, then the regularized luminosity $f(z)$ is constant.

Example (constant regularized luminosity):

suppose we have measured a constant regularized luminosity $f(z) \equiv L H_{0}^{2} /\left(4 \pi c^{2}\right)$. Then the Hubble rate is

$H=(z+1) \sqrt{H_{0}^{2}(z+1)^{2}-k c^{2} z^{2} / a_{0}^{2}}$.

The three solutions of these three differential equations, $k=$ $\pm 1,0$, in terms of the scale factors are obtained from Eqs. (13) and (14):

$$
\begin{aligned}
& a(t)= a_{0}\left(2 H_{0} t\right)^{1 / 2} \sqrt{1-k\left[\frac{1-\left(2 H_{0} t\right)^{1 / 2}}{a_{0} H_{0} / c}\right]^{2}}, \\
& 0<t<t_{0}=\frac{1}{2 H_{0}} .
\end{aligned}
$$

To alleviate notations, we have suppressed the subscripts $\cdot_{ \pm}$ from $a(t)$ and $a_{0}$. In the closed case, $a_{0}$ must satisfy the inequality (12):

$a_{0} \geq \frac{c}{H_{0}} \frac{z_{\max }}{z_{\max }+1}$

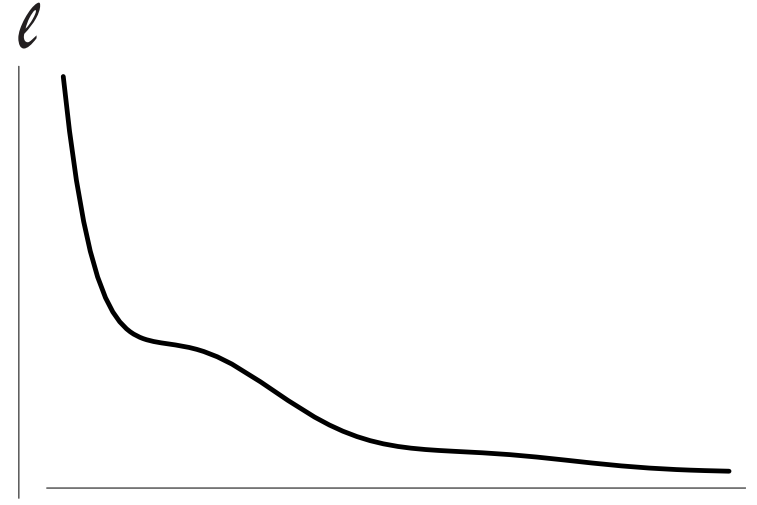

$z$

Fig. 1. The monotonic luminosity (22).

and the inflection point of $g(z)$ is hidden behind the horizon at $\chi=\arcsin \left[c /\left(a_{0} H_{0}\right)\right]$.

Example (constant Hubble rate):

to have an example without a horizon, consider the scale factor

$a(t)=a_{0} \exp \left[H_{0}\left(t-t_{0}\right)\right]$.

This is a limiting case of the first example with $p \rightarrow 1$. It has constant Hubble rate and constant deceleration parameter, $H(z) \equiv H_{0}, q(z) \equiv-1$, and $\chi=c z /\left(a_{0} H_{0}\right)$. In the closed case, $g(z)$ has an infinite number of inflection points alternating with short distance divergencies. The inflection points are all minima, with the first located at $z_{\text {infl }}=\pi a_{0} H_{0} /(2 c)$.

Example (closed universe): suppose we have measured the luminosity:

$\ell(z)=\frac{L H_{0}^{2}}{4 \pi c^{2}} \frac{z+2}{z^{2}}$.

It is strictly decreasing and has the correct short distance divergency. Its $g(z)$ has one and only one inflection point at $z_{\text {infl }}=(1+\sqrt{17}) / 2 \sim 2.56$, which suggests a closed universe with $a_{0} \sim 0.337 \mathrm{c} / \mathrm{H}_{0}$.

Counter-example (wiggling $g(z)$ ):

suppose we have measured the luminosity, Fig. 1:

$\ell(z)=\frac{L H_{0}^{2}}{4 \pi c^{2}} \frac{(\sin z / z)^{2}+0.1}{z^{2}}$.

Again it is strictly decreasing and has the correct short distance divergency. Now $g(z)$ has a maximum, Fig. 2. Therefore no Robertson-Walker universe, neither open nor closed, exists with this luminosity.

The last two examples illustrate that our constraint of monotonic $g(z)$ is stronger than the constraint of monotonic luminosity $\ell(z)$.

\section{Non-monotonic scale factors}

If the scale factor is strictly decreasing, we get similar results with a negative spectral deformation: $-1<z<0$, "blue shift". One might think that one can produce non-monotonic functions $g(z)$ by starting from non-monotonic scale factors $a(t)$, 


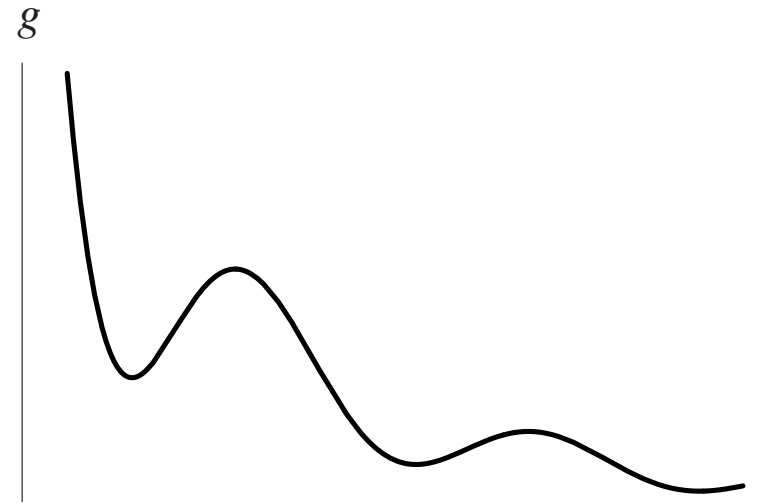

Fig. 2. Its wiggling $g(z)$.

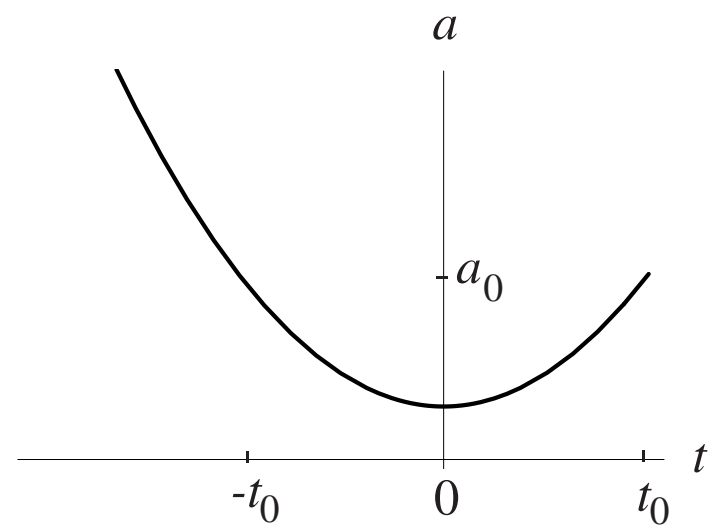

Fig. 3. The non-monotonic scale factor (23).

but this is not true. In fact any non-monotonic scale factor produces multi-valued luminosities in terms of the spectral deformation $z$. The first example is, of course, the constant scale factor with no spectral deformation, $z \equiv 0$, but varying luminosity. A more generic example is shown in Fig. 3:

$a(t)=\frac{1}{2} g t^{2}+\alpha, \quad g, \alpha>0$.

For positive $t_{0}$, we have a maximal redshift of $z_{\max }=a_{0} / \alpha-1$. We have $z=0$ for $t= \pm t_{0}$ and a blue shift for $t<-t_{0}$ :

$-1<z_{\min }=\frac{a_{0}}{\frac{1}{2} g t^{2}+\alpha}-1<0$.

The dimensionless co-moving distance is

$\chi(t)=c \sqrt{\frac{2}{g \alpha}}\left[\arctan \left(\sqrt{\frac{g}{2 \alpha}} t_{0}\right)-\arctan \left(\sqrt{\frac{g}{2 \alpha}} t\right)\right]$

and the relation between emission time $t$ and spectral deformation $z$ is

$t(z)= \pm \sqrt{\frac{2}{g}} \sqrt{\frac{a_{0}}{z+1}-\alpha}$.

Note that the Hubble rate vanishes at the inflection time $t=$ $0, z=z_{\max }$, while the deceleration parameter diverges there. Also the regularized luminosity vanishes at $t=-t_{0}, z=0$. As $t$ tends to minus infinity, $z$ tends to -1 and the luminosity, regularized or not, diverges. We call this phenomenon the

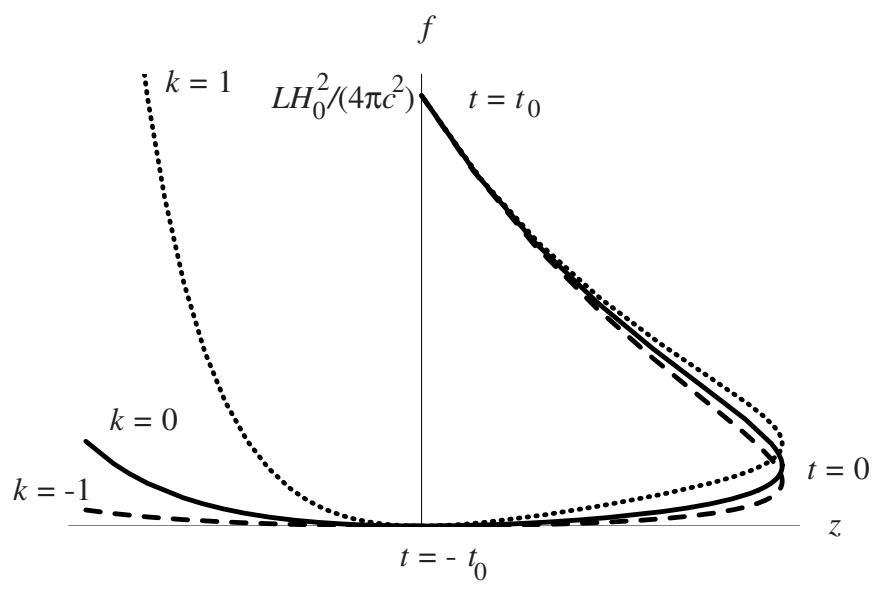

Fig. 4. The regularized luminosity of the non-monotonic scale factor (23)

"ultra-violet divergency". In the closed case, we have in addition antipode and short distance divergencies. Figure 4 shows only one antipode singularity.

\section{Data and conclusion}

We use the "Gold" sample data compiled by Riess et al. (2004) with 157 SN's, including a few at $z>1.3$ from the Hubble Space Telescope (HST GOODS ACS Treasury survey). For convenience we normalize the luminosity to the maximum absolute SN luminosity estimated by Jha et al. (1999), Saha et al. (2001), and Gibson \& Stetson (2001), $L=L_{\max }=$ $(1 \pm 0.1) \times 10^{35} \mathrm{~W}$. The Hubble rate today $H_{0}$ is taken as $(70 \pm 5) \mathrm{km} \mathrm{s}^{-1} \mathrm{Mpc}^{-1}$ (Krauss 2001; Raux 2003).

The regularized luminosity allows us to extract $L H_{0}^{2}$ from the Hubble diagram with small red shift, Fig. 5. The value $f(0)$ is extracted by a second order polynomial extrapolation fit on the SN data up to a red shift of 0.1. By construction, the fitted value $f(0)$ is equal to $L H_{0}^{2} /\left(4 \pi c^{2}\right)=(4.3 \pm 0.4) \times 10^{26} \mathrm{~W} \mathrm{Mpc}^{-2}$, where the error is only coming from the fit itself.

\subsection{Lower bound on the radius of the universe today}

The first of Eqs. (9) and Eq. (12) together give the lower bound on $a_{0}$ as

$a_{0} \geq \sqrt{\frac{f(0)}{g_{\min }}} \frac{c}{H_{0}}$

The minimal value of $g(z)$ is obtained from the $\mathrm{SN}$ recorded at $z=1.3$ and is equal to $g_{\min }=(3.83 \pm 0.72) \times 10^{26} \mathrm{~W} \mathrm{Mpc}^{-2}$. At a $95 \%$ confidence level (CL), we have the upper bound on the minimal value of $g(z): g_{\min }<5.6 \times 10^{26} \mathrm{~W} \mathrm{Mpc}^{-2}$. Combining the errors from $f(0)$ and $g_{\min }$ gives the lower bound on $a_{0}$ at 95\% CL:

$a_{0}>0.88 c / H_{0} \sim 3.8 \mathrm{Gpc} \sim 1.2 \times 10^{26} \mathrm{~m}$

$\sim 1.3 \times 10^{10}$ light years. 


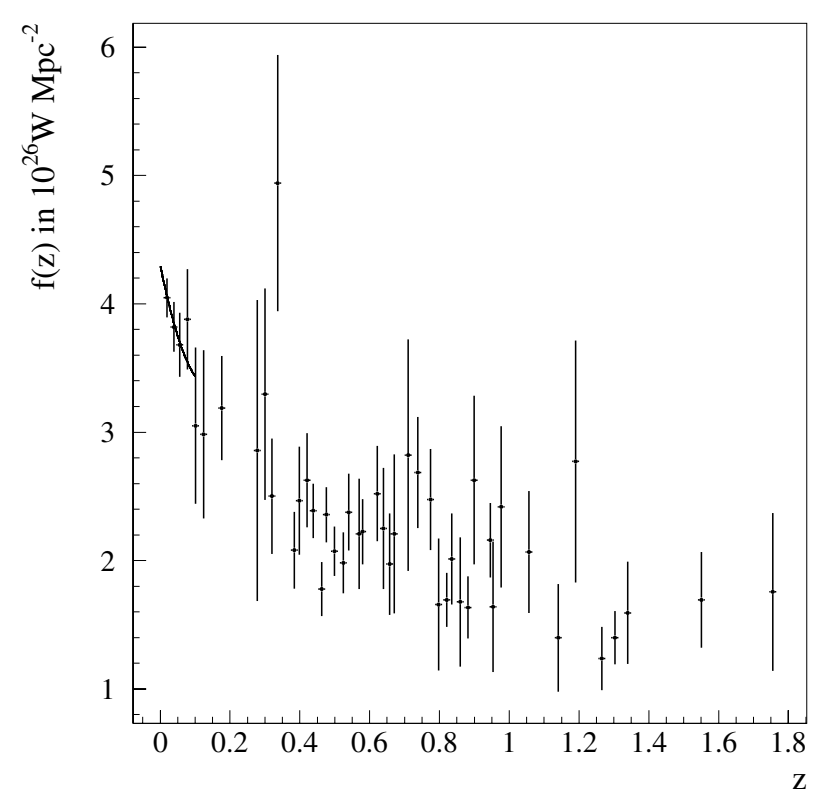

Fig. 5. The regularized luminosity as measured today (Riess et al. 2004), with a binning of 0.02 in red shift. The full line at low red shift corresponds to a second order polynomial extrapolation fit.

This lower bound on $a_{0}$ translates into a lower bound on the curvature density,

$\Omega_{k 0}:=-k\left(\frac{c}{a_{0} H_{0}}\right)^{2}>-1.29$ at $95 \% \mathrm{CL}$.

Note that this limit is independent of the values of the absolute luminosity $L$ and of the Hubble rate today. It is also independent of any dynamical hypothesis.

Let us compare this bound with the one obtained from the $\mathrm{SN}$ data but now adding the dynamics of the $\Lambda C D M$ cosmology fitting the matter density $\Omega_{\mathrm{m}}$, the cosmological constant density $\Omega_{\Lambda}$, and the nuisance normalization parameter, but without any other input constraint: $\Omega_{k 0}>-1$ at $95 \%$ CL.

Including $\gamma$ ray bursts with their high $z$ potentially improves our bound, but does not do so at present because of the high uncertainty of their absolute luminosity. Also, we expect a small improvement on our bound from SNAP data: $a_{0}>1.04 c / H_{0}$, $\Omega_{k 0}>-0.91$ at $95 \% \mathrm{CL}$. This improvement relies on the assumption that the central values of the apparent luminosities will not change significantly and comes from the reduction of the error bars, mainly due to adding more nearby supernovae (Yèche et al. 2006).

Of course our bound from supernova data cannot compete with constraints from CMB anisotropy data. It has, however, the virtue of not being subject to any dynamical hypotheses, like a cosmological constant, dark matter, exotic equations of state, power spectra, inflation, etc.

\subsection{Wiggles and non-monotonicity}

We must now ask the question whether the data is compatible with a monotonic luminosity $\ell(z)$. We will also ask the finer question of whether $g(z)$ is monotonic.
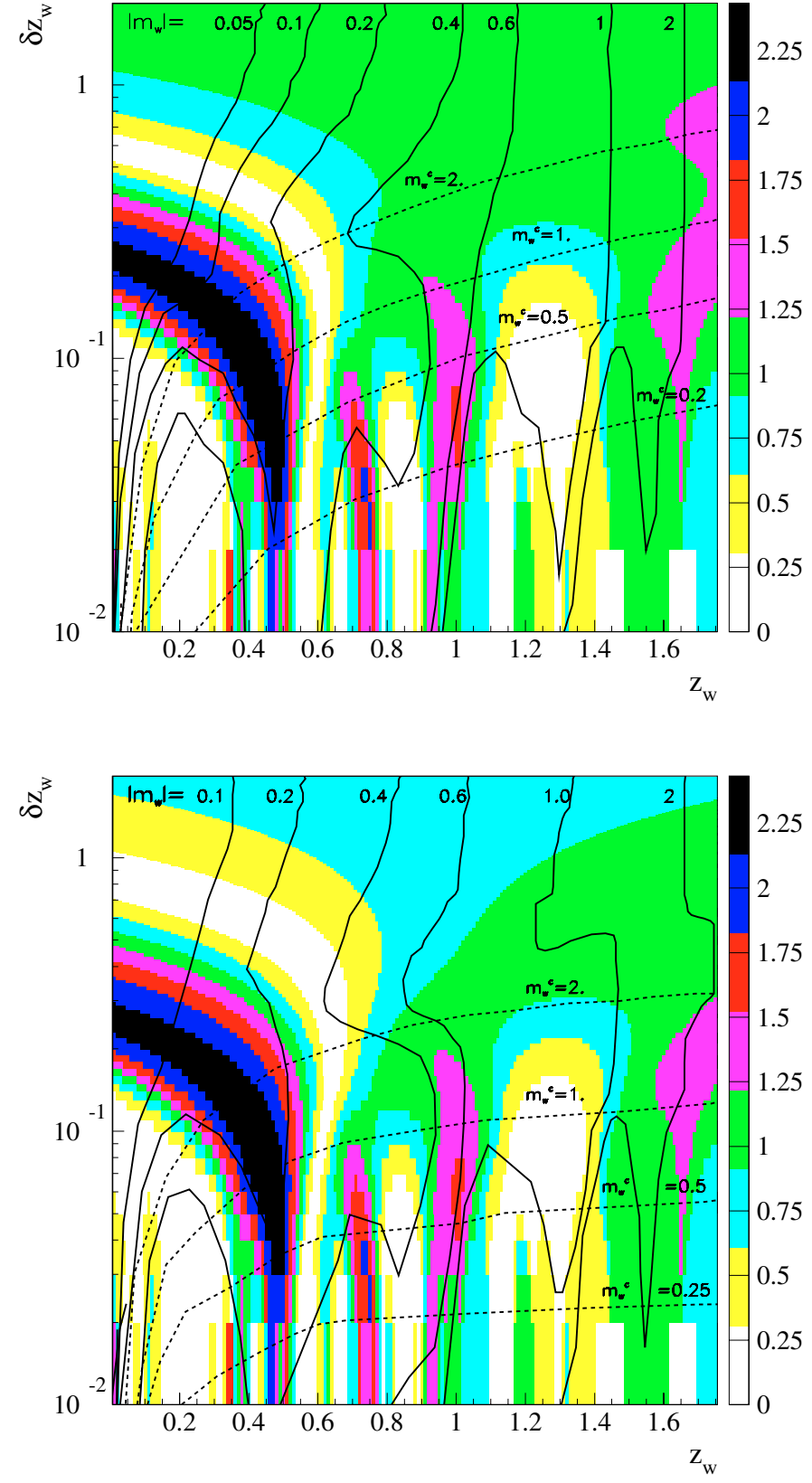

Fig. 6. Color contours: significance of wiggle detection (vertical colour scale) as function of the wiggle position $z_{\mathrm{w}}$ and width $\delta z_{\mathrm{w}}$, logarithmic scale. Full lines: expected $2 \sigma$ sensitivity of the wiggle detection as a function of wiggle magnitude $m_{\mathrm{w}}$. The dashed lines indicate the location of the critical wiggle magnitude $m_{\mathrm{w}}^{\mathrm{c}}$ of a positive wiggle that breaks the monotonicity. The upper panel is the exclusion plot for a non-monotonic luminosity $\ell(z)$, the lower panel for nonmonotonic $g(z)$.

To detect non-monotonicity in the SN data set, we assume that the luminosity $\ell(z)$ and its $g(z)$ can be described by monotonic functions to which we add a simple Gaussian:

$L(z)=\ell\left(z, L H_{0}^{2}, p\right)+a_{\mathrm{w}} \exp -\left(z-z_{\mathrm{w}}\right)^{2} /\left(2 \delta z_{\mathrm{w}}^{2}\right)$

and

$G(z)=g\left(z, L H_{0}^{2}, p\right)+a_{\mathrm{w}} \exp -\left(z-z_{\mathrm{w}}\right)^{2} /\left(2 \delta z_{\mathrm{w}}^{2}\right)$. 
The monotonic functions $\ell\left(z, L H_{0}^{2}, p\right)$ and $g\left(z, L H_{0}^{2}, p\right)$ are derived from a power law parameterization of the scale factor $a(t):=a_{0}\left(p H_{0} t\right)^{1 / p}$ (constant deceleration parameter), $1 / 5<$ $p<1, k=0$. It yields the monotonic luminosity

$\ell\left(z, L H_{0}^{2}, p\right)=\frac{L H_{0}^{2}}{4 \pi c^{2}}\left(\frac{p-1}{(z+1)\left[1-(z+1)^{1-p}\right]}\right)^{2}$,

and with $p=0.69$ it describes the $\Lambda C D M$ cosmology well up to a redshift of 1.8 (Henry-Couannier et al. 2005). Its acceleration is positive with $q \equiv-0.31$.

Our wiggle detection procedure consists of scanning the plane in wiggle position $z_{\mathrm{w}}$ and wiggle width $\delta z_{\mathrm{w}}$ in steps of 0.01 in both directions. In each point $\left(z_{\mathrm{w}}, \delta z_{\mathrm{w}}\right)$ of this plane, we fit the normalization $L H_{0}^{2}$, the power $p$ and the wiggle amplitude $a_{\mathrm{w}}$. Warning: if the wiggle amplitude $a_{\mathrm{w}}$ is smaller than a critical amplitude $a_{\mathrm{w}}^{c}$ the modified functions (30) and (31) will still be monotonic.

We could claim that $\ell(z)$, and all the more the luminosity $g(z)$, is not monotonic if the ratio between the fitted wiggle amplitude and the associated error is greater than $5(5 \sigma$ level detection) and if the wiggle amplitude is greater than the critical one. The sensitivity of the method is computed by Monte Carlo simulation. The same SN sample as the Riess data set with the same statistical power is simulated by assuming the $\Lambda \mathrm{CDM}$ cosmology, and a wiggle of positive or negative amplitude is added to $\ell(z)$ and $g(z)$ for each point in the $\left(z_{\mathrm{W}}, \delta z_{\mathrm{w}}\right)$ plane. We apply the wiggle detection procedure to each simulation, restricted to a small grid of points around the simulated one to speed the processing up. The significance of the wiggle amplitude $\left.\left(\mid a_{\mathrm{w} \text { fitted }}\right) \mid / \sigma_{a_{\mathrm{w}}}\right)$ is computed at each point, and the smaller value from the positive or negative wiggle amplitude is retained. The sensitivity is computed at a $2 \sigma$ level corresponding to a $95 \%$ confidence level exclusion limit on the wiggle magnitude $m_{\mathrm{w}}$ defined by

$a_{\mathrm{w}}\left(z_{\mathrm{w}}\right)= \pm\left(10^{-m_{\mathrm{w}} / 2.5}-1\right) \ell\left(z_{\mathrm{w}}\right)$.
Figure 6 shows the significance of the wiggle fit performed on the Riess data sample (color contours) for the luminosity $\ell(z)$ and $g(z)$ with $z_{\mathrm{w}}$ varying from 0.01 to 1.8 and $\delta z_{\mathrm{w}}$ from 0.01 to 2 in steps of 0.01 in both directions. The maximum significance for both $\ell(z)$ and $g(z)$ is 2.4 for a wiggle at the position $z_{\mathrm{w}}=0.45$, with width of 0.07 . The wiggle magnitude is $m_{\mathrm{w}}=0.16$ for luminosity $\ell(z)$, and $m_{\mathrm{w}}=0.25$ for $g(z)$. The dashed lines indicate the location of the critical wiggle magnitude $m_{\mathrm{w}}^{\mathrm{c}}$ of a positive wiggle that breaks the monotonicity.

No wiggle greater than this value is observed, so we conclude that no wiggle is detected at a $5 \sigma$ level using the actual $\mathrm{SN}$ data set. On the same figures, the sensitivity for different values of the wiggle magnitude is shown (plain line). Wiggles of magnitude greater than 2 are excluded at 95\% CL up to a redshift of 1.6. Up to a redshift of 1 , the 95\% CL exclusion limit on the wiggle magnitude is 0.6 . These two magnitudes are below the critical magnitudes; therefore, these wiggles do not upset the monotonicity.

\section{References}

Berry, M. 1976, Principles of Cosmology and Gravitation (Cambridge: Cambridge University Press)

Esposito-Farèse, G., \& Polarski, D. 2001, Phys. Rev. D, 63, 063504

Gibson, B. K., \& Stetson, P. B. 2001, ApJ, 547, L103

Henry-Couannier, F., et al. 2005, gr-qc/0507065, in press

Jha, S., Garnavich, P. M., Kirshner, R. P., et al. 1999, ApJS, 125, 73

Krauss, L. M. 2001, Space, time and matter: Cosmological Parameters, in Identification of Dark Matter

Raux, J. 2003, Photométrie différentielle de supernovae de type Ia lointaines $(0.5<z<1.2)$ mesurées avec le télescope spatial Hubble et estimation des paramètres cosmologiques, Ph.D. Thesis, Université Paris 11

Riess, A. G., Strolger, L.-G., Tonry, J., et al. 2004, ApJ, 607, 665

Saha, A., Sandage, A., Tammann, G. A., et al. 2001, ApJ, 562, 314

Yèche, C., et al. 2006, A\&A, in press [arXiv: astro-ph/0507170] 